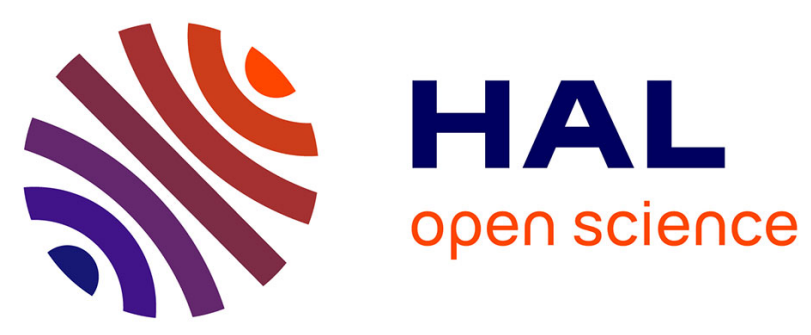

\title{
ELIPSE: An Innovative Technology for the Treatment of Radioactive Organic Liquids
}

\author{
F. Lemont, M. Marchand, M. Mabrouk, D. Milelli, J.-M. Baronnet
}

\section{To cite this version:}

F. Lemont, M. Marchand, M. Mabrouk, D. Milelli, J.-M. Baronnet. ELIPSE: An Innovative Technology for the Treatment of Radioactive Organic Liquids. Nuclear Technology, 2017, 198, pp.53-63. 10.1080/00295450.2017.1289009 . cea-02421886

\section{HAL Id: cea-02421886 https://hal-cea.archives-ouvertes.fr/cea-02421886}

Submitted on 20 Dec 2019

HAL is a multi-disciplinary open access archive for the deposit and dissemination of scientific research documents, whether they are published or not. The documents may come from teaching and research institutions in France or abroad, or from public or private research centers.
L'archive ouverte pluridisciplinaire HAL, est destinée au dépôt et à la diffusion de documents scientifiques de niveau recherche, publiés ou non, émanant des établissements d'enseignement et de recherche français ou étrangers, des laboratoires publics ou privés. 


\section{ELIPSE - AN INNOVATIVE TECHNOLOGY FOR THE TREATMENT OF RADIOACTIVE ORGANIC LIQUIDS}

Florent LEMONT ${ }^{(\mathrm{a})}$, Mickael Marchand ${ }^{(\mathrm{a})}$, Majdi Mabrouk ${ }^{(\mathrm{a})}$, Doriana Milelli ${ }^{(\mathrm{a})}$, Jean Marie Baronnet ${ }^{(\mathrm{b})}$

(a) CEA, DEN, DTCD, SCDV, LPIC, F-30207 Bagnols-sur-Cèze, France

(b) University of Limoges, GRESE 123 Avenue Albert Thomas, 87060 Limoges, France

Contact: $\quad$ Florent LEMONT, CEA, DEN, DE2D, SEVT, LPTI, F-30207 Baglons-sur-Cèze

Florent.lemont@cea.fr

Number of pages: 25

Number of tables: 1

Number of Figures: 15 


\section{ABSTRACT}

The CEA is studying the development of processes with a sufficiently large operating range in order to propose a general treatment system and make it possible to absorb a significant quantity of radioactive liquid stocks awaiting treatment around the world. A solution may be the use of submerged plasma into which the organic liquids would be injected. Current research has demonstrated that such a technique may enable the instantaneous and complete destruction of liquids with a wide variety of constituants, such as chlorine, fluorine or phosphorus. The ELIPSE process was designed based on the results of this research. In this process, an arc plasma torch is submerged in the core of an aqueous solution. The submersion solution offers many advantages: quenching and cleaning of combustion gases; filtering of the particles they contain; and cooling maintained for the entire process, which guarantees excellent corrosion control. An advantage of this type of design is that the gas treatment system can be reduced to a demister-condenser followed by a simple safety filter, thereby offering the additional advantage of an extremely compact treatment system. This design also allows the ELIPSE process to become by this way an embeddable process if required.

The present paper will first describe the state-of-the-art concerning this concept and secondly research currently conducted using the ELIPSE process to destroy a wide variety of liquids such as TBP, trichloroethylene and perfluoropolyether with an efficiency of over $99 \%$ at rates of several liters per hour. The apparent absence of any corrosion observed in the treatment system would indicate that, following optimization, a universal and compact process may soon be available, which may be transportable and dedicated to the treatment of orphan waste products awaiting treatment.

\section{Keywords: ELIPSE process, submerged plasma, liquid treatment}




\section{CONTEXT AND RAPID OVERVIEW OF STATE OF THE ART}

\section{I.A Organic liquids and plasma processes}

Organic liquids, or liquids made up of a portion of organic compounds, represent a significant quantity of radioactive waste stored at various nuclear sites around the world [1]. The treatment processes for these liquids depend on their composition and their activity. For example, low or very low level short-lived liquids are generally destroyed in traditional incineration processes such as Socodei [2] when their chemical compositions are compatible with the acceptance criteria for the treatment technology. Higher activity liquids, either due to the fact that the radioelements they contain have longer lives or that they contain levels of compounds such as chlorine or fluorine that exceed the maximum process limit, must be treated using the appropriate technologies, which are often exclusive. For example, extraction solvent waste with high levels of phosphorus resulting from fuel reprocessing activities is treated via dedicated processes at its operating site [3-4]. Many other waste straems, produced in varying quantities at nuclear sites or various research laboratories, represent an extremely varied stock awaiting a treatment solution. For example, in the UK there is more than $600 \mathrm{~m}^{3}$ of contaminated oils [5]. Generally, the radioactive activities of the liquids, their volumes and compositions which vary according to their origin make them difficult to treat. In the latter case, they are referred to as orphan waste, and are stored pending the existence of an appropriate disposition path.

When the liquids are organic, the solutions adapted to their treatment must take into account the existence of a component that must be properly destroyed. Thermal processes which have proven to be good candidates for ensuring their treatment must, in the design phase, anticipate risks of corrosion or other harmful phenomena due to the presence of aggressive chemical elements such as chlorine, sulfur, phosphorus, fluorine, etc. Amongst these processes, those making use of plasma torches are the preferable solution due to their ability 
to generate extremely high temperatures and therefore to destroy, with acceptable kinetics, liquids that are not easily degradable. Many of these processes were developed at the end of the 1980s in order to comply with the requirements of the Montreal protocol relating to the destruction of chemical compounds responsible for the depletion of the ozone layer [6].

Certain processes developed for the treatment of solid waste may be used for some LLW liquids which have a composition that is compatible with the technological structures. This is the case, for example, with the plasma systems developed by the company Retech [7], in which the materials to be treated are placed in a centrifugal crucible which guarantees uniform heat treatment and differentiated flow of the various types of melted materials. Other processes, such as SHIVA (currently under development) [8], may also be useful outlets for ILW (Intermediate Level Waste) liquids. As for plasma systems developed specifically to treat liquids, there are relatively few on the market. The only plasma system that is actually marketed is the Plascon process developed in Australia in the 1990s [9]. It uses an arc plasma in which the liquid to be processed is vaporized. The gases containing reaction products are quenched in order to limit the possibility of any recombination that may result in the production of dioxins or furans. These applications are currently used only for hazardous—not radioactive-liquids.

The rapid gas quenching concept, as used in the Plascon process, led to the idea of placing the plasma as close as possible to the solutions to be treated, or to the solutions for cleaning the combustion gases.

\section{I.B Development of submerged plasma}

The first studies conducted in this area were mainly focused on evaluating the capability of a submerged plasma to produce $\mathrm{HO}$ or $\mathrm{O}$ radicals, or even ozone, in a solution. The idea was then to determine the means for decontaminating aqueous solutions contaminated by organic pollutants. In the 1980s, Alekseev et Al. [10-11] studied heat transfer from a plasma to the aqueous solution in which it is submerged, and estimated that the quenching rate for 
gases is approximately $10^{7} \mathrm{~K} \cdot \mathrm{s}^{-1}$. Extremely high speeds such as these made it possible to fix the radicals capable of oxidizing the organic compounds present in the water.

It was based on this phenomenon that certain processes were proposed as possible decontamination solutions. Bernier et al. [12], for example, propose a concept using an arc plasma torch submerged in a solution to be decontaminated. Figure 1 shows a schematic representation of the process, and indicates an effective decrease in cyanide decontamination over time. The curve shows that the use of $10 \mathrm{~kW}$ plasma results in a reduction of approximately $90 \%$ within 2 hours.
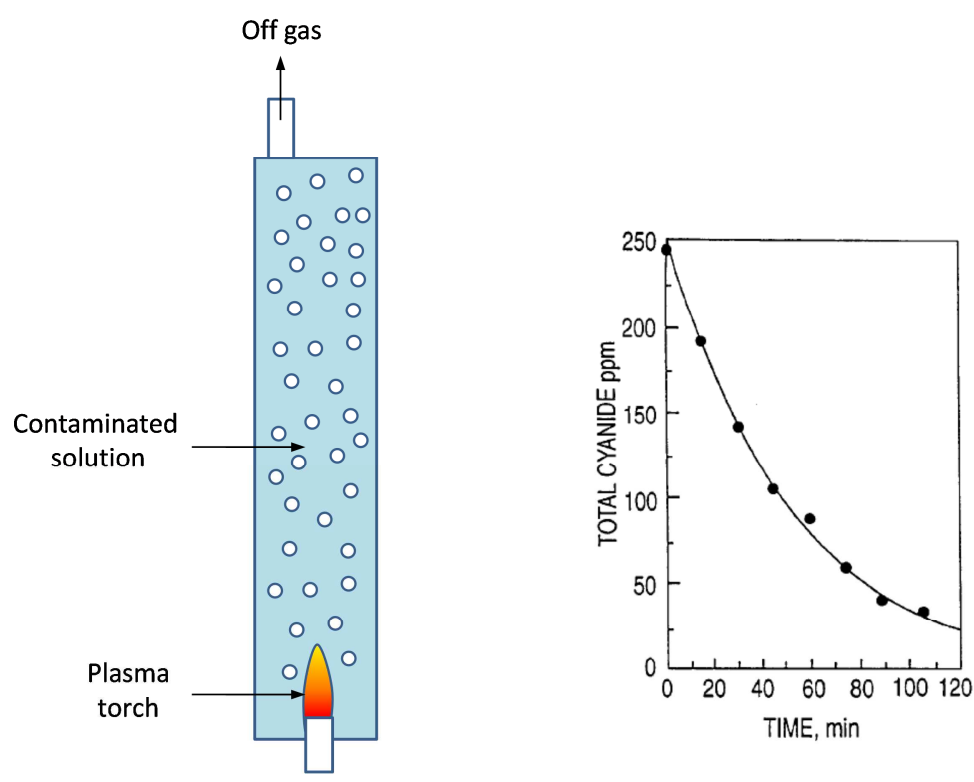

Figure 1: Schematic representation of a process using a submerged plasma torch (left). Decontamination kinetics of cyanide solution (right).

Although this concept can be used to treat aqueous solutions, it cannot be used for the treatment of pure organic liquids. Other processes have therefore been developed to propose suitable outlets. Uchiyama et al. [13], for example, propose a system which is quite representative of what has been proposed by others. It uses a technology which destroys organic liquids in a combustion chamber, where the output is atomized directly in an aqueous solution (Figure 2). A system was designed to treat flow rates of approximately $1 \mathrm{~L}^{-1} \mathrm{~h}^{-1}$ for waste solvents (TBP-Dodecane) used in fuel treatment operations. 
Studies of this type of process show that the combustion is not truly submerged, as a combustion chamber with refractors is used. The heated zone which generates the combustion reactions and gas transit is therefore quite large, and subject to the aggressive nature of the combustion gases which contain heteroatoms that may cause corrosion. The range of liquids that could be treated in this type of structure is therefore quite small.

Processes have also been developed by JAEA in Japan [14] for the treatment of TBP/dodecan. JAEA has two main processes for this application, a steam reforming process and a submerged combustion process. In the second case, the liquid mixture is supplied at a rate of $1-3 \mathrm{~kg} \mathrm{~h}^{-1}$ to a gasification chamber where it is vaporized and pyrolyzed (Figure 3). The decomposition gases are then completely burned in a submerged combustion reactor to prevent soot and dioxins generation.

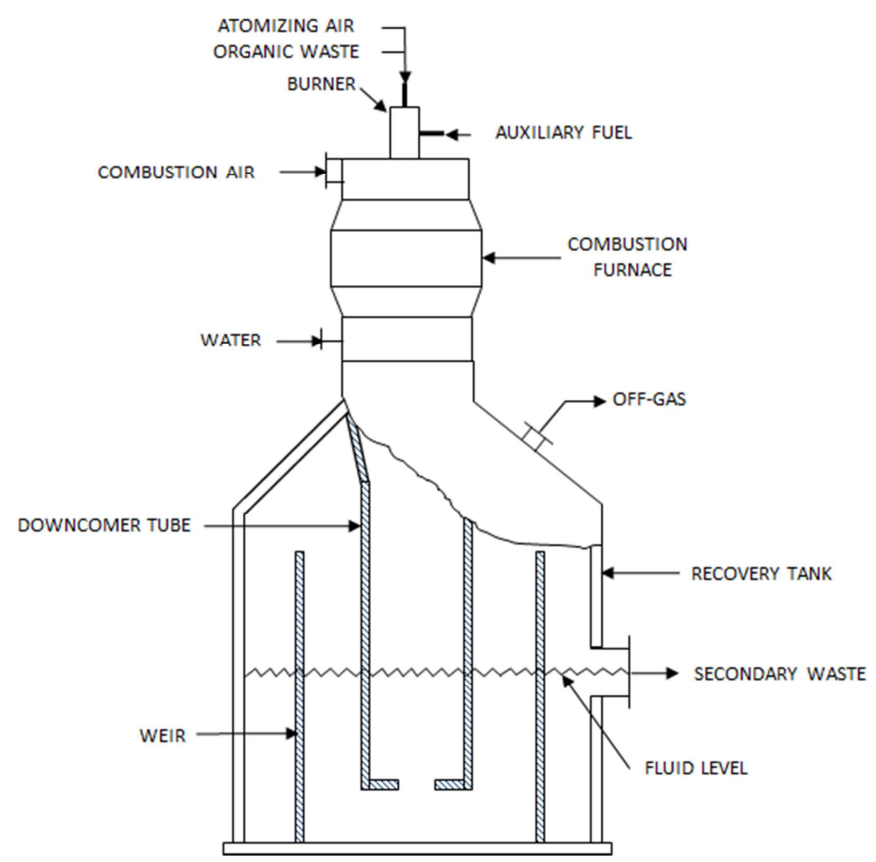

Figure 2: Process using "submerged" combustion

In this latest example, what is called "submerged combustion" concerns only the afterburner and not the whole thermal treatment of the waste. Corrosion may then dramatically occur in the hot zone. 


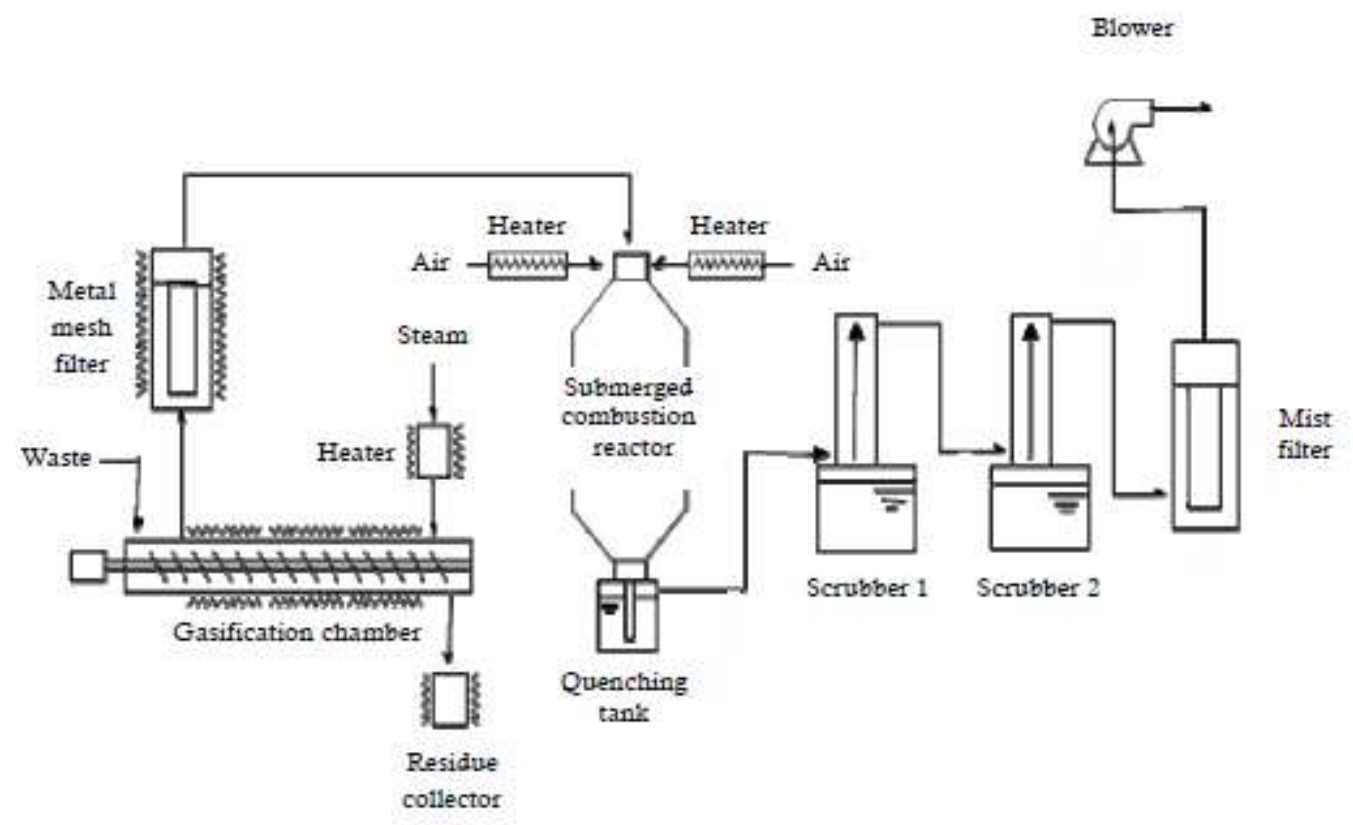

Figure 3: Gasification chamber followed by a "submerged combustion reactor" by JAEA

Faced with the need to treat liquids in different quantities and with highly varied compositions, the CEA has undertaken a research program to combine the two types of technology described above.

\section{THE ELIPSE PROCESS}

\section{II.A General description}

The ELIPSE process has been developed to treat liquids with extremely varied physicochemical properties and compositions. The goal is to find an effective outlet for all or a portion of "orphan" liquids stored at industrial centers or research laboratories. Nonexhaustive inventories have shown that these liquids are classified under the categories of lubricants, solvents, thinners, decontamination agents and extraction agents [15] with mineral or heteroatom contents that make them difficult to treat with a single process. For low or mid level radioactive liquids, it is in fact quite difficult to consider a single process capable of treating TriButylPhosphate (TBP) containing high levels of phosphorus, 
TriChloroEthylene(TCE) containing high levels of chlorine, or perfluorinated oil such as Fomblin containing high levels of fluorine using the same unit, which must withstand the corrosive nature of these contents.

ELIPSE, which uses submerged plasma in which the liquids are destroyed, offers a solution to this problem because the process is fully cooled, and therefore much less sensitive to corrosion.

The process, which is shown on the left-hand side of Figure 4, includes an arc plasma torch placed in the bottom of a reactor filled with water. The organic liquids to be treated are then injected in a highly reactive oxygen plasma where they are fully oxidized.

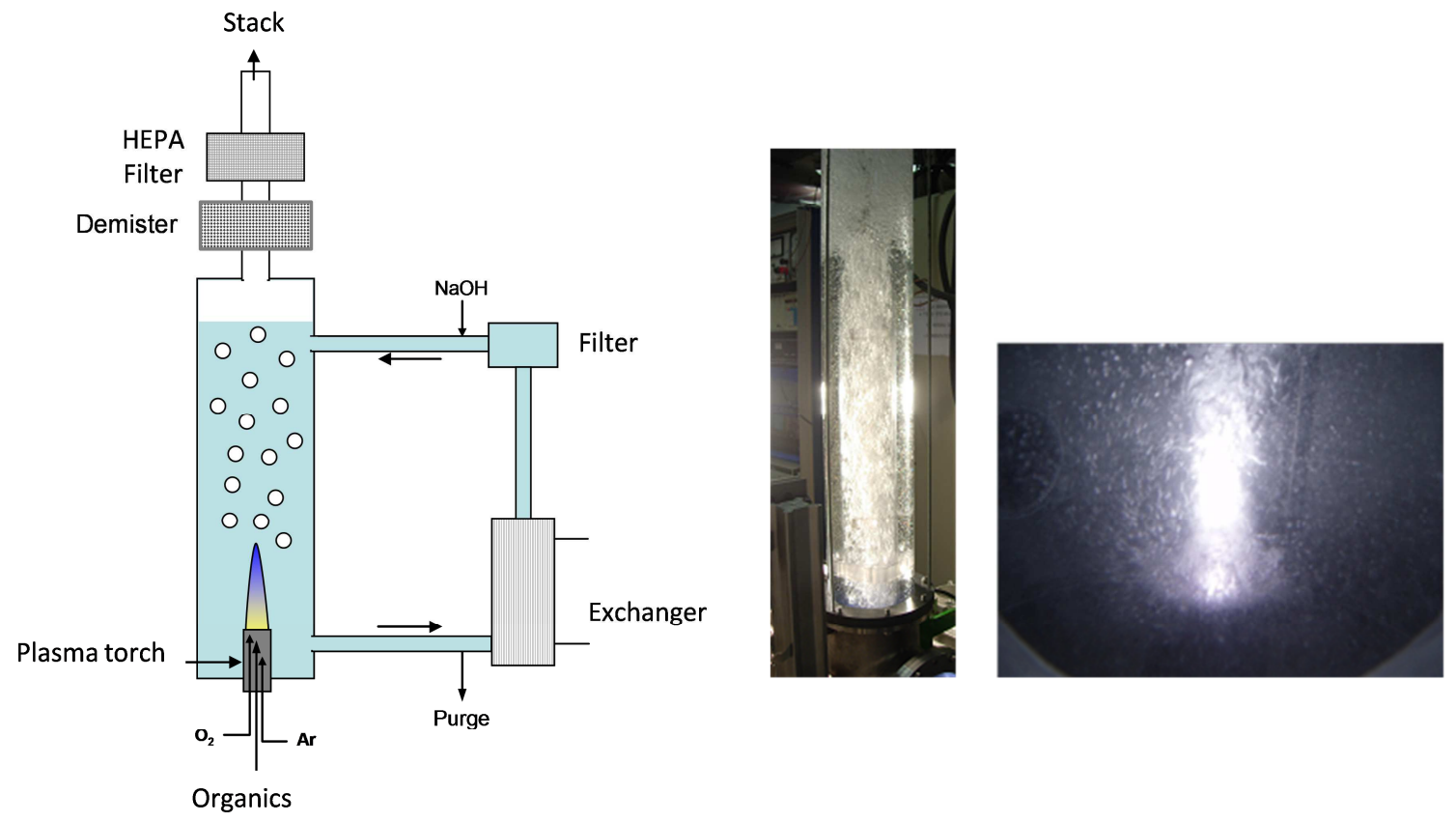

Figure 4: Left: Diagram of ELIPSE process. Right: Photograph of plasma working in water.

The water contained in the reactor is continuously filtered and cooled via a circulation system that includes an exchanger, a filter and a system which maintains the $\mathrm{pH}$ at the required operating level.

The combustion gases pass through a water column and then through a condenser-demister to eliminate the droplets and water vapor. A High-Efficiency Particulate Arresting (HEPA) 
filter guarantees total arresting of solid particulates in cases where some of the particulates are not retained in the aqueous solution of the reactor.

The gas treatment system is limited to the condenser-demister and the HEPA filter, which explains the compact nature of the treatment assembly because the water column contained in the reactor ensures the following:

- $\quad$ Cooling of the combustion gases, with subsequent heat transfer to the exchanger on the circulation system.

Filtering of combustion gases. The mineral contents contained in the organic waste are retained in suspension in the water and then recovered by the filtering system in the circulation loop.

- $\quad$ Scrubbing of gases. The dissolved and solvated chemical species in the aqueous solution are continuously neutralized via a pre-set $\mathrm{pH}$ control system. Adjustments are made by adding base products, which may be caustic soda or any other soluble hydroxide.

Filter and condenser-demister implemented in the mockup are commonly used in industry.

\section{II.B Plasma torch, injection of organic liquid and combustion}

The process uses an arc plasma torch with approximately $50 \mathrm{~kW}$ generated between a tungsten cathode and a copper anode. The cathode is shielded by argon gas flow of around $30 \mathrm{NL} \cdot \mathrm{min}^{-1}$ (NL for Normal Liter), and oxygen is injected downstream to form reactive plasma $\left(\approx 200 \mathrm{NL} \cdot \mathrm{min}^{-1}\right)$. The flow rates of each gas are measured by mass flowmeter. The plasma exits from the top of the anode, which is followed by a part for organic liquid injection (see Figure 5). The temperatures obtained in the plasma core cannot be measured, but are sufficiently high for the oxygen to be considered totally separated in its elemental form. It is then possible, by considering the nature of the gaseous flow as described above and the energy injected in the plasma (approximately $30 \mathrm{~kW}$ ), to calculate the specific enthalpy of the

gases, which is approximately $5.8 \mathrm{~kJ}^{-1}$. The estimated value for the temperature is deduced on the curve showing the changes in enthalpy per mass of the $\mathrm{Ar} / \mathrm{O}_{2}$ system based 
on temperature. In the present case, and as shown on Figure 5, it reaches approximately $3500 \mathrm{~K}$.

Each component of the plasma torch is therefore subject to extremely high temperatures, and their cooling by internal water circulation (not shown on Figure 5) was therefore actually implemented.
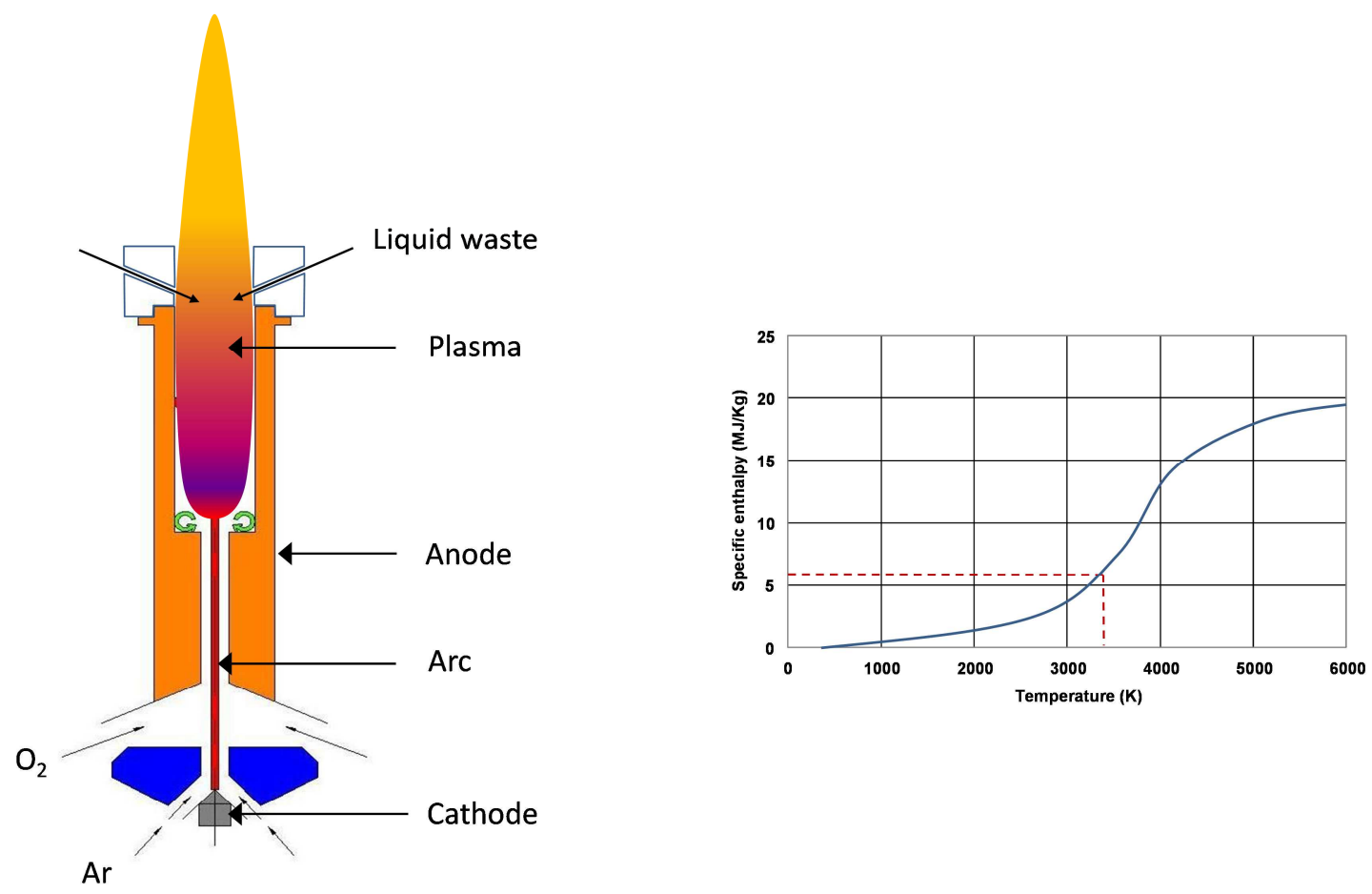

Figure 5: Left: Diagram of plasma torch installed beneath an organic liquid injection component. Right: Specific enthalpy/temperature relationship in plasma.

When the organic liquid reaches the plasma, it is immediately vaporized and oxidized. The combustion gases are then cooled to a lower temperature to displace the $\mathrm{CO} / \mathrm{CO}_{2}$ equilibrium towards a zone rich in $\mathrm{CO}_{2}$ to prevent the production of combustible gases [16]. This thermal displacement is performed by two additional parts shown in Figure 6:

The first part, which is referred to as the dilution stage, performs water injection to quickly and efficiently reduce the temperature of the gases via vaporization.

- $\quad$ The second, which is referred to as the cooling stage, ensures a sufficient resident time for the gases so that chemical equilibrium can be reached. It is made up of a cylinder with cooled walls which lower the gas temperature of approximately $200 \mathrm{~K}$. 


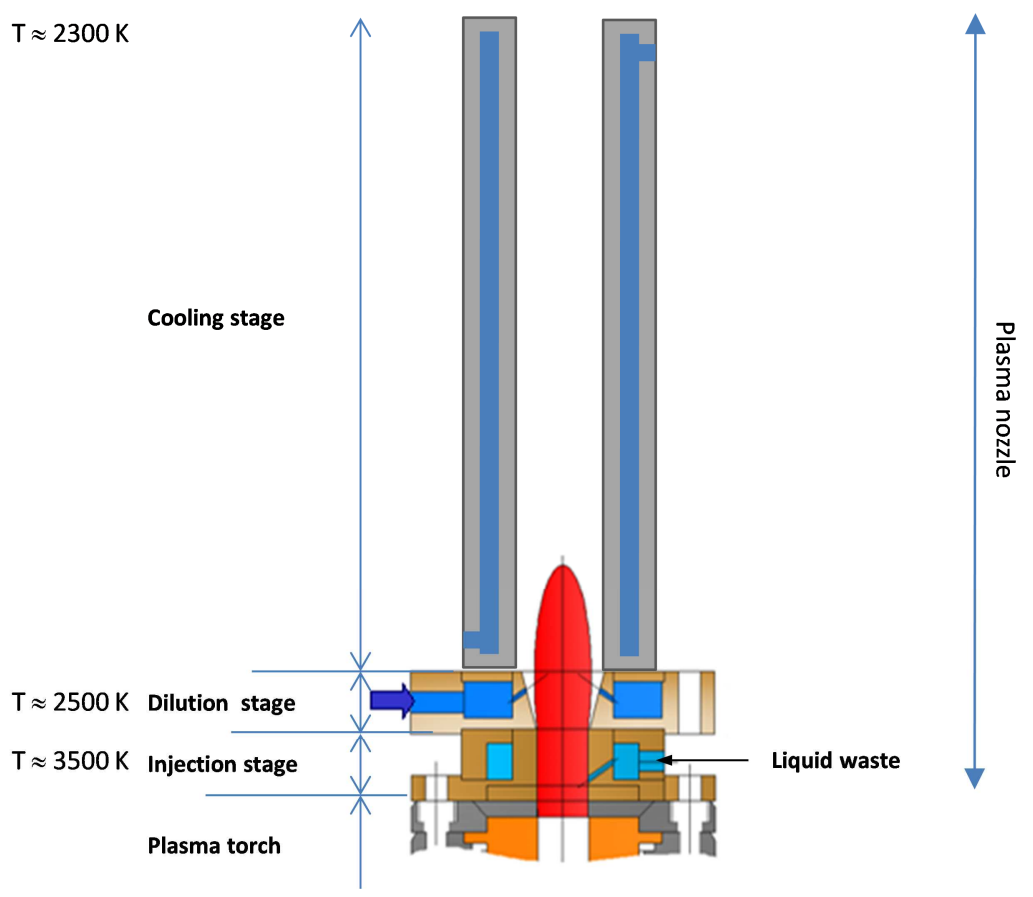

Figure 6: Diagram of plasma torch nozzle in reactor

The total volume of the torch nozzle, which is approximately $59 \mathrm{~cm}^{3}$, ensures a resident time for the gases of approximately $1.3 \mathrm{~ms}$, which is sufficient to guarantee optimal oxidization of the injected organic materials. In the configuration described above, the gases enter the reactor solution at a temperature of approximately $2300 \mathrm{~K}$ and a rate of $250 \mathrm{~ms}^{-1}$.

Each of the three nozzle components described above is made of Inconel 601 cooled by internal water circulation in order to keep corrosion problems to a minimum.

\section{II.C Experimental pilot facility}

Based on the conclusive results of the laboratory tests, a pilot facility was built and installed at the Marcoule research center. This equipment, which is shown in Figure 6, was designed to treat liquids at rates exceeding 3 L.h ${ }^{-1}$. Windows were included in its construction in order to observe what was happening inside the reactor in operation. 
In the foreground of the left-hand picture in Figure 7 is the reactor equipped with four viewing windows. The plasma torch placed in the bottom of the reactor can be seen in the right-hand picture of Figure 7. A condenser-demister was installed directly on its top section to reduce outgoing water to the gas extraction system. In the background of the picture on the left can be seen the reactor's water circulation loop, equipped with a filter, an exchanger, a pH probe with a caustic soda injection pump, and a set of temperature probes and flowmeters used to perform heat balance assessments required to optimize the process. All the part of the process (except plasma torch and its nozzle) is made of stainless steel $316 \mathrm{~L}$ for a better corrosion resistance.
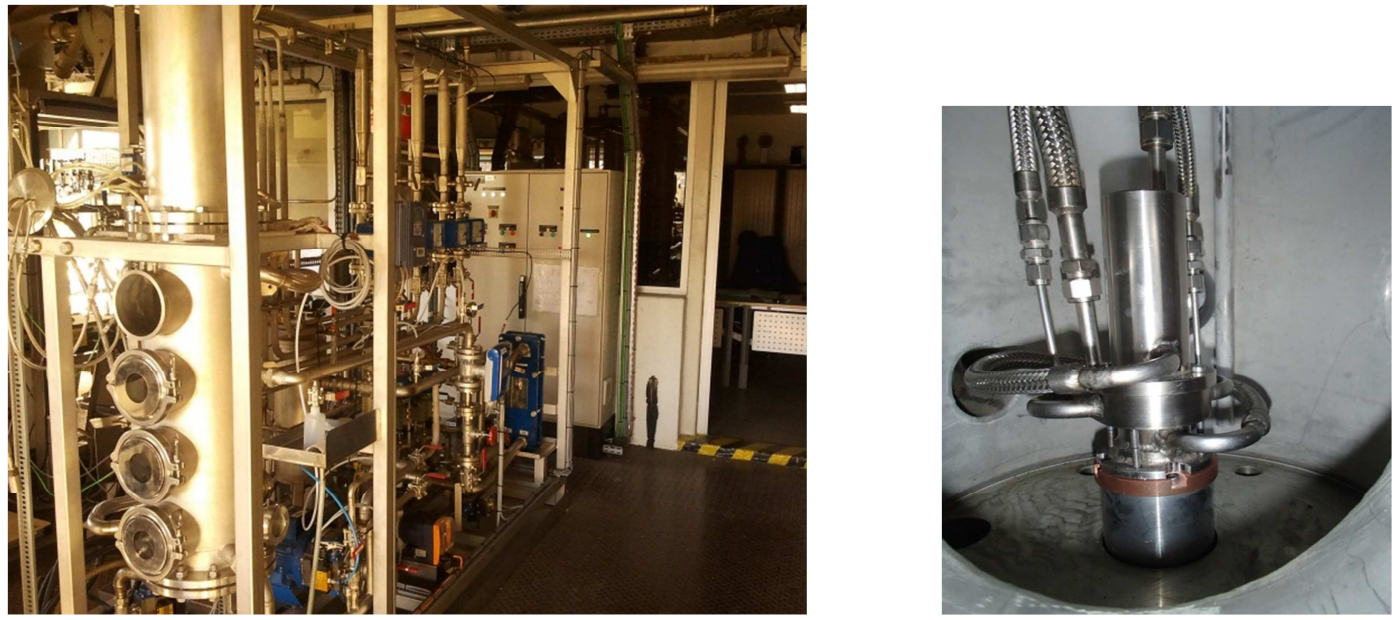

Figure 7: Left: ELIPSE installed at Marcoule. Right: Plasma torch installed in bottom of reactor.

\section{OPERATION AND PERFORMANCES}

\section{III.A General case}

The organic liquid to be destroyed, which can be represented in a simplified version as $\mathrm{C}_{m} \mathrm{H}_{n} \mathrm{O}_{p} \mathrm{X}_{\mathrm{r}}$, is introduced in the plasma nozzle as described above. The combustive oxygen, present in an ionized and/or radical form, quickly oxidizes the compound, which is transformed into gaseous combustion products that may or may not be soluble depending on the case. The mineral content initially contained in the liquid is transferred in the aqueous solution of the reactor in a precipitated, complexed or solvated form. Depending on the 
obtained destruction rate, a residual organic compound (quantity $\varepsilon$ ) may also be transferred to the core of the aqueous solution. According to the schematic diagram of the chemical process shown in Figure 8, the destruction rate $\rho$ for the process may be expressed as follows:

$\rho=1-\frac{\varepsilon}{q}$

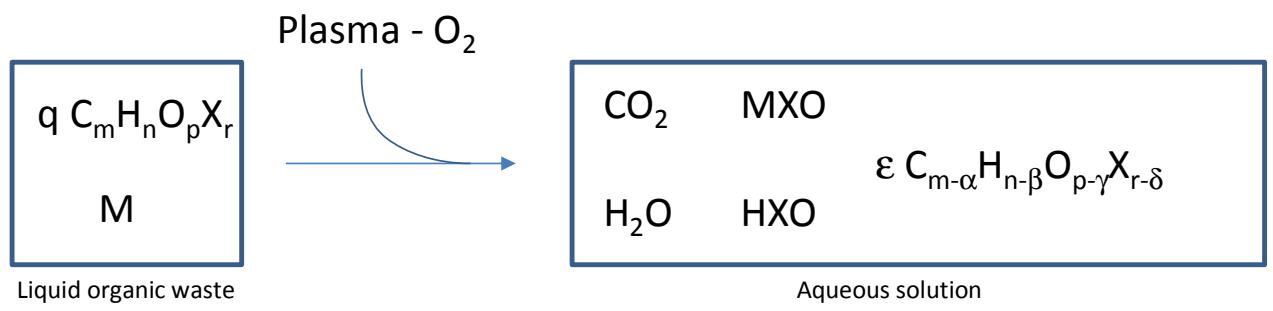

Figure 8: Schematic diagram of oxidized destruction of an organic liquid containing mineral $M$ in the ELIPSE process

where q corresponds to the quantity of organics fed in the plasma and MXO corresponds to the oxidized mineral content, which may be related to the heteroatom entering into the composition of the organic compound. HXO corresponds to the hydrogenated, and in certain cases oxidized, heteroatom. It is processed in the core of the cooled aqueous solution and continuously treated by a dedicated loop.

\section{III.B Circulation and neutralization system}

When the organic liquid is destroyed in the plasma torch nozzle, a portion of the power generated by the plasma and the combustion is transferred to the water, which increases in temperature. The mineral contents will also be transferred in the water in forms that will depend on which chemical species are present, the temperature, and the $\mathrm{pH}$ of the solution.

Due to the transfers between the plasma nozzle and the aqueous solution, the solution must continuously be treated for filtering, cooling, and neutralization. As was mentioned earlier, the solution is continuously treated by a system containing the following: 
- $\quad$ A pH probe which controls the supply pump for the caustic soda, or for another neutralizing element

- A filter to separate and recover solid mineral contents from the solution. In the experimental ELIPSE mockup, the filter pore dimension can vary from $1 \mu \mathrm{m}$ up to $50 \mu \mathrm{m}$

- $\quad$ A plate heat exchanger which maintains the solution at a temperature below $40^{\circ} \mathrm{C}$

- A circulation pump for the fluid at a rate of approximately $5 m^{3} \cdot h^{-1}$

An intake valve which sends a portion of the solution to the dilution stage of the plasma nozzle

Figure 9 shows the accompanying system for the reactor.

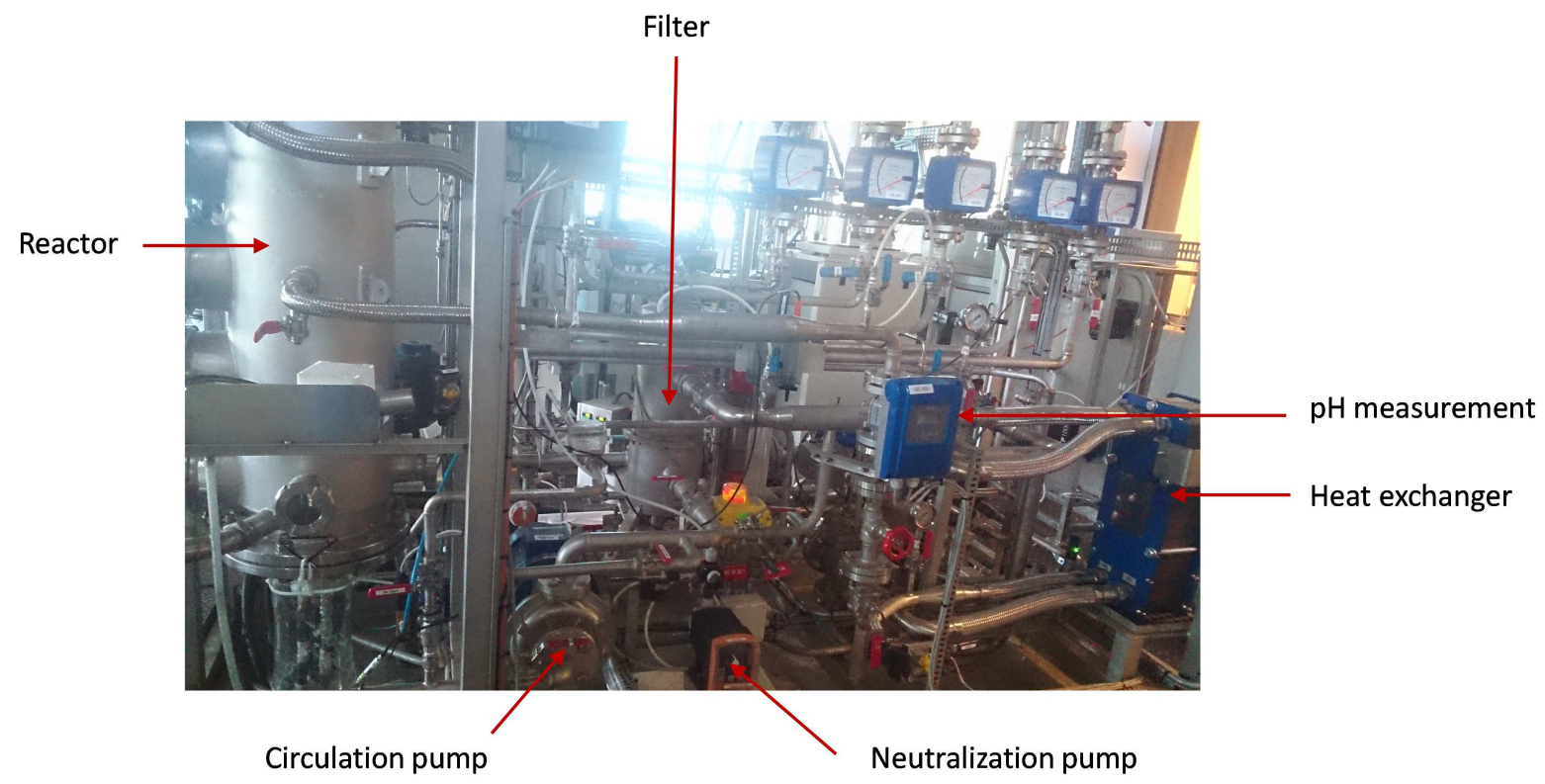

Figure 9: Treatment system for solution line on reactor

All of the equipment installed on the pilot facility is standard equipment that has been widely validated and qualified for multiple industrial applications. Their configuration is optimal for their intended functions. 
The $\mathrm{pH}$ adjustment is a fundamental aspect, as the trapping and speciation of the species in the solution depend on it. Trapping of $\mathrm{CO}_{2}$, which is the most predominant gaseous species, must be avoided in order to prevent rapid saturation of equipment, and in particular the filter for carbonates. Based on the diagram of species proportions shown in Figure 10-A, the $\mathrm{pH}$ of the solution should ideally be less than 4 so that the $\mathrm{CO}_{2}$ remains in a gaseous form. The upper limit for this $\mathrm{pH}$ is, according to the diagram, approximately 8 . Above this $\mathrm{pH}$, all of the $\mathrm{CO}_{2}$ remains trapped as carbonates.

In addition, it is essential to maintain the heteroatoms from the liquid in their basic form in order to limit their corrosive properties. For example, if we consider the treatment of TBP/Dodecane based on the reaction (2) described in the following paragraph, phosphoric acid $\left(\mathrm{H}_{3} \mathrm{PO}_{4}\right)$ is continually produced in the solution, and its form will also depend on the $\mathrm{pH}$ as indicated in the species proportion diagram shown in Figure 10-B. The need to neutralize the phosphoric acid means it is necessary to work with a $\mathrm{pH}$ that is greater than 4 , thereby requiring the presence of hydrogen carbonate in the solution based on Figure 10-A. What's more, the solubility of the residue-in particular of hydrogen phosphate, which is the predominant species-will depend on the neutralizing reagent. Neutralization with caustic soda results in the production of the compound $\mathrm{Na}_{2} \mathrm{HPO}_{4}$, with a solubility of $77 \mathrm{gl}^{-1}$ at $20^{\circ} \mathrm{C}$, whereas neutralization with lime results in the production of the compound $\mathrm{CaHPO}_{4}$ with a solubility of $0.2 \mathrm{gl}^{-1}$. Furthermore, caustic soda produces the compound $\mathrm{NaHCO}_{3}$ with a solubility of $87 \mathrm{gl}^{-1}$ at $20^{\circ} \mathrm{C}$, whereas lime produces the compound $\mathrm{Ca}\left(\mathrm{HCO}_{3}\right)_{2}$ with a solubility of $166 \mathrm{gl}^{-1}$ at $20^{\circ} \mathrm{C}$. The filter operations will depend on thes e differences in solubility, as clogging will occur more or less rapidly depending on the selected operating mode and duty point. 


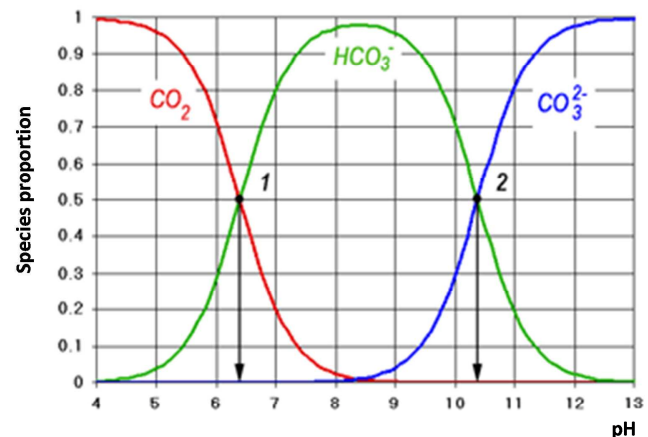

A

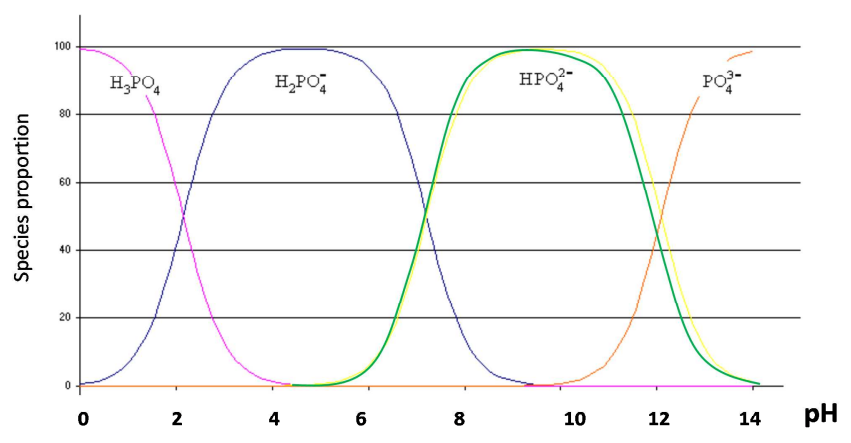

B

\section{Figure 10: Speciation of carbon $(A)$ and phosphorous $(B)$ in solution based on $p H$.}

The neutralization must be much more finely adjusted for the treatment of fluorinated liquids, considering that in this case, it is necessary to rapidly precipitate all of the fluorine to avoid free form HF. Neutralization with lime that produces the compound $\mathrm{CaF}_{2}$ with a very low solubility $\left(0.015 \mathrm{gl}^{-1}\right)$ is then the recommended choice for a $\mathrm{pH}$ more than one decade higher

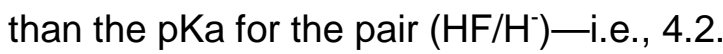

For the treatment of complex mixtures containing multiple heteroatoms, it may be preferable to set the operating range at a $\mathrm{pH}$ which is close to 10 in order to ensure total neutralization of all the species. In this case, the $\mathrm{CO}_{2}$ will be totally converted into carbonate and then be processed by the final containment system.

\section{III.C Application for TBP-Dodecane extractant}

If the organic liquid is the extractant TBP-Dodecane containing traces of uranium that have not been re-extracted in the form uranyl nitrate, the overall calculation for the destruction of one liter of mixture with the molar ratio of 0.9 mole of TBP for 3.3 moles of Dodecane $(70 \%$ weight of Dodecane) may be proposed in the following format:

$0.9 \mathrm{C}_{12} \mathrm{H}_{27} \mathrm{O}_{4} \mathrm{P}+3.3 \mathrm{C}_{12} \mathrm{H}_{26}+\zeta \mathrm{UO}_{2}\left(\mathrm{NO}_{3}\right)_{2}+77.25 \mathrm{O}_{2} \rightarrow 50.4 \mathrm{CO}_{2}+53.7 \mathrm{H}_{2} \mathrm{O}+0.9 \mathrm{H}_{3} \mathrm{PO}_{4}+2 \zeta \mathrm{HNO}_{3}+\zeta \mathrm{UO}_{2}$ 
In this example (not totally balanced with additional $2 \zeta \mathrm{H}$ coming from water for $\mathrm{NO}_{3}$ protonation and $\zeta O$ coming from oxygen plasma), the speciation of uranium remains to be studied, as it may be freed in oxide form after the uranyl has passed through the plasma. It may also be freed in the form of uranium phosphate, or eventually be solvated as uranium nitrate.

From a thermal point of view, the oxidation of the mixture will dissipate a total energy that is close to the standard reaction enthalpy because the mixture is introduced at ambient temperature, and the reaction products at the temperature of the solution in the reactor. The enthalpy for the TBP formation is estimated at $1457.1 \mathrm{~kJ} . \mathrm{mol}^{-1}$ [17], and the calculation for the overall reaction enthalpy (2) corresponding to 1 liter of solution is approximately $-33819 \mathrm{~kJ}$. It can easily be deduced that the dissipated power for a rate of 1 L. $\mathrm{h}^{-1}$ of mixture will be approximately $9.4 \mathrm{~kW}$. In general, for the treatment of any given liquid, the power developed in the system will be the power injected by the plasma $\left(\mathrm{P}_{\text {Plasma }}\right)$, plus the power exchanged during the treatment of 1 liter per hour of liquid $\left(\Delta \mathrm{H}_{\mathrm{r} / \mathrm{L}}\right)$ multiplied by overall processing rate $\mathrm{D}$ :

$P_{t}=P_{\text {plasma }}+\frac{\Delta H_{r / L}}{3600} \mathrm{D}$

The dissipated power will be evacuated via the various process cooling systems, including all the internal circulation lines of the torch and its nozzle, the cooled reactor walls, the condenser-demister and the heat exchanger on the process water circulation loop.

The curve shown in Figure 11 represents the power balance for a test conducted with a plasma torch supplied with $52 \mathrm{~kW}$ of stabilized electrical power, and in which different feeding rates of TBP-Dodecane mixture were used. These experiments have been performed with simply chemical without radioelements. The feeding rates are provided by a calibrated peristaltic pump and checked thanks to the monitoring of the mass of the supply cylinder. The red curve represents the power injected into the torch over time; the black curve represents the total dissipated power in all the cooling systems, measured with the test 
instrumentation and shown at the top of Figure 8. The difference naturally represents the power dissipated in the torch by combustion of the organic liquid feed.

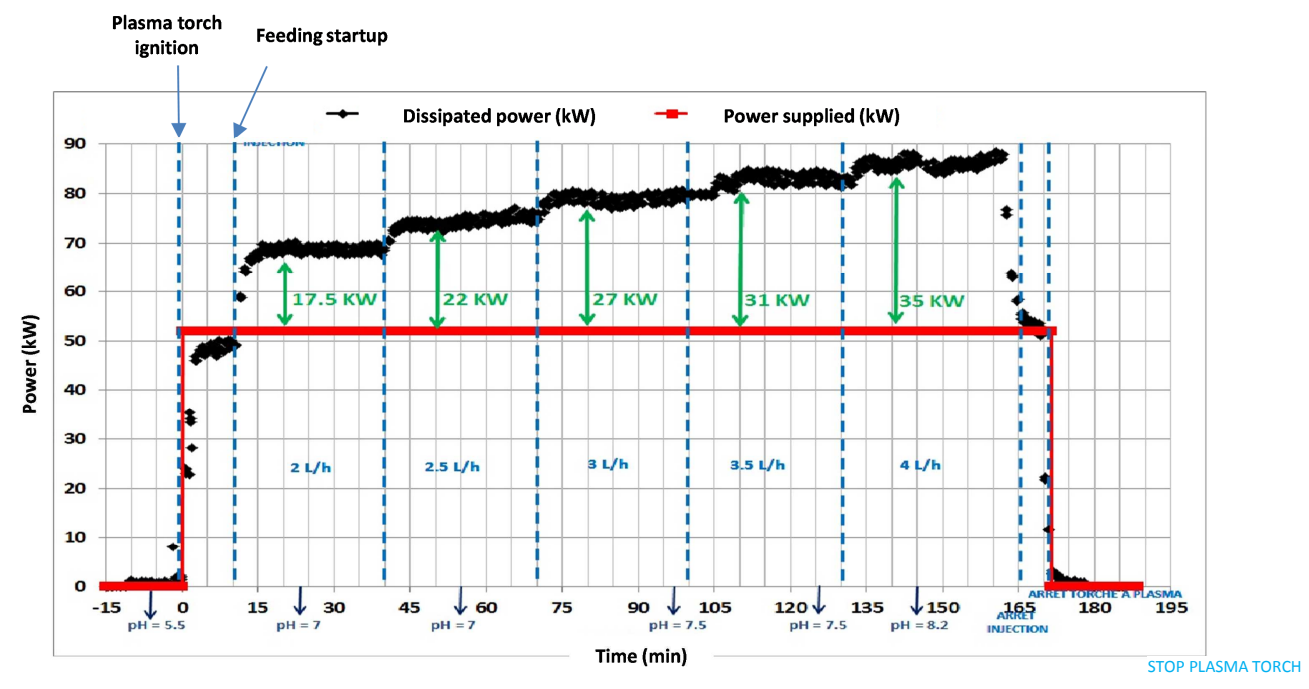

STOP INJECTION

Figure 11: Power balance during treatment of TBP-Dodecane mixture at variable feeding rates.

The deviations measured between the red curve and the black curve for different feed rates correspond to the power dissipated by the combustion of the organic mixture. Figure 12 below shows the close correspondence between the theoretical power calculated based on the equation (3) and the measurement taken on the treatment facility.

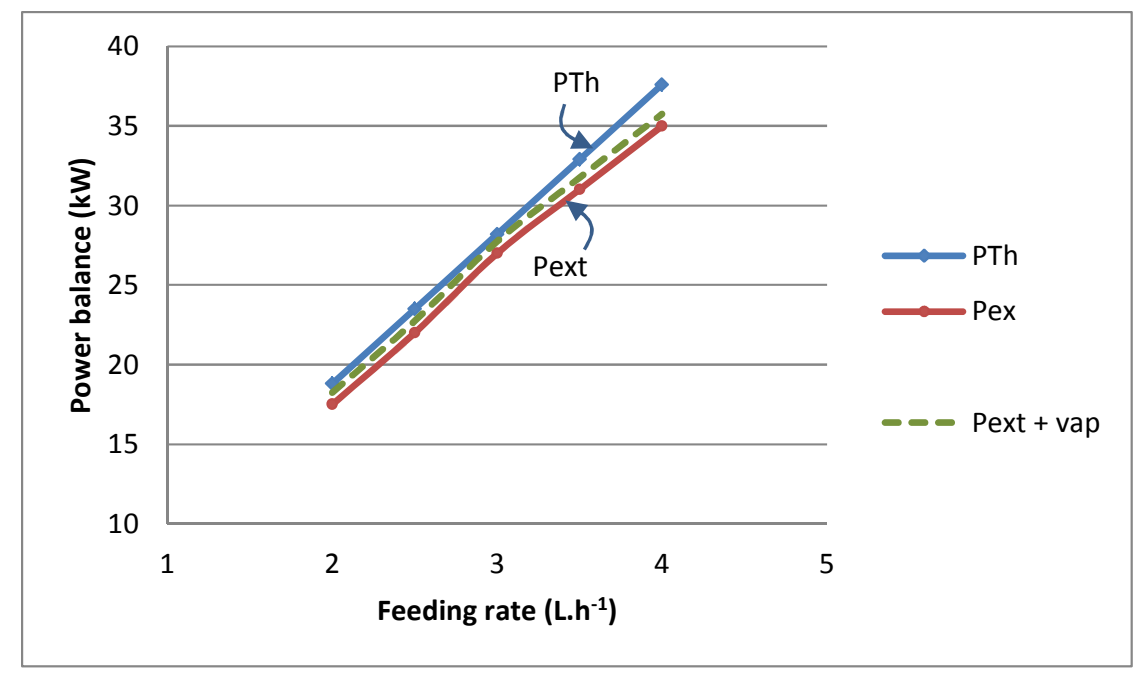

Figure 12: Comparison between theoretical power dissipated during treatment with variable TBP-Dodecane feeding rate and the measured power. 
A hygrometry measurement of the gases at the condenser outlet shows a relative humidity of $100 \%$, representing a total water loss for the 155 minutes of treatment of approximately $2.5 \mathrm{~L}$ (quantity measured after performing a material balance). Considering the enthalpy of the water vaporization $\left(2675 \mathrm{~kJ} \mathrm{~kg}^{-1}\right)$ applied to this quantity and the treatment time, it appears that this continuous process absorbs approximately $0.75 \mathrm{~kW}$, which provides an even more accurate power balance as shown in Figure 12.

These thermal balances, which demonstrate the excellent efficiency of the experimental pilot facility, made it possible to evaluate its capability of destroying the three categories of liquids described above.

\section{III.D Overall performance}

To evaluate the potential of ELIPSE to treat a wide variety of liquids, its effectiveness for the destruction of 3 compounds cited in the general process description was investigated:

- The TBP/Dodecane mixture widely used in the nuclear industry as an extractant in the PUREX retreatment process. The thermal power generated by its oxygen combustion is $9.4 \mathrm{~kW} \cdot \mathrm{h} \cdot \mathrm{L}^{-1}$.

- Trichloroethylene (TCE), a slightly flammable solvent whose use is now prohibited for safety reasons. The thermal power generated by its oxygen combustion is $3.0 \mathrm{~kW} \cdot \mathrm{h} \cdot \mathrm{L}^{-1}$.

- A perfluoropolyether (PFPE) product from Fomblin® used as a lubricant in UF $_{6}$ transfer pumps. The thermal power generated by its oxygen combustion is $4.8 \mathrm{~kW} \cdot \mathrm{h} \mathrm{L}^{-1}$.

The respective molecules are shown in Figure 13. 


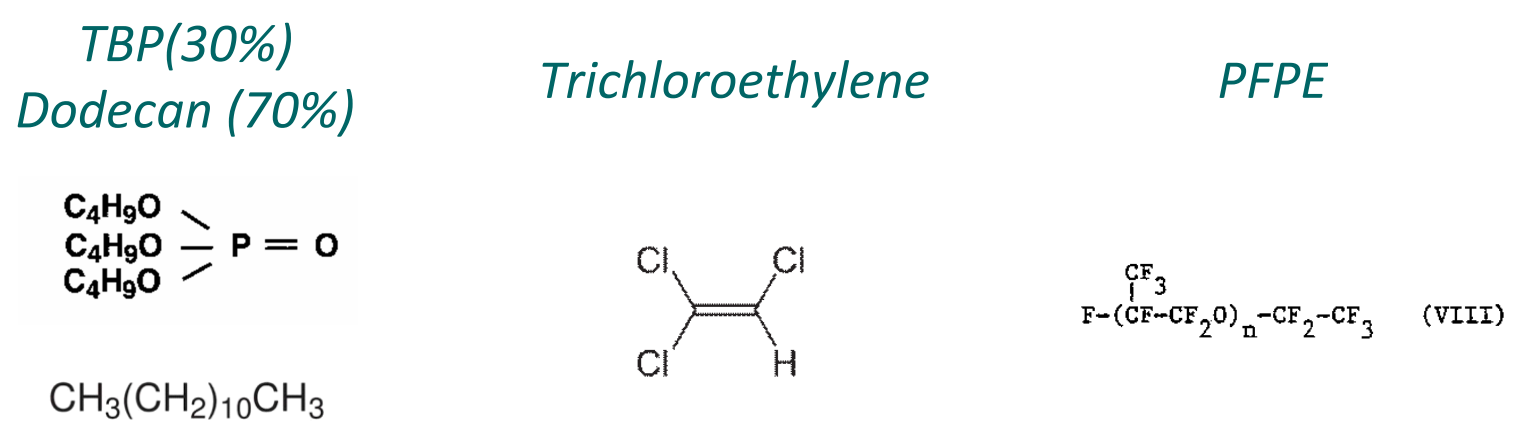

Figure 13: 3 molecules with varying charges undergoing ELIPSE treatment.

The treatment of each liquid was initially evaluated at a treatment rate of $2{\mathrm{~L} \cdot \mathrm{h}^{-1}}$ for 1 hour, in the operating mode specified in the general process description.

The destruction efficiency was evaluated by analyzing the Total Organic Carbon (TOC) in the residual solution. The TOC is measured with a SHIMADZU analyzer, model TOC-L, using the $680^{\circ} \mathrm{C}$ combustion catalytic oxidation method. The analysis time for the device is between 3 and 7 minutes, and the detection limit is between $0.5 \mu \mathrm{g} / \mathrm{L}$ and 20,000 mg/L.

The destruction efficiency is calculated using the following equation:

$\eta_{d}=\frac{C_{i}-C_{f}}{C_{i}}$

where $C_{i}$ is the TOC introduced in the process and $C_{f}$ is the final TOC analyzed at the end of treatment.

The results obtained using this method are indicated in table 1.

\begin{tabular}{|c|c|c|c|}
\hline Liquid & TBP/Dodecan & TCE & PFPE \\
\hline$\eta$ & $99.90 \%$ & $99.99 \%$ & $99.80 \%$ \\
\hline
\end{tabular}

Table 1: Destruction efficiencies obtained for different liquids

The post-test inspection performed on the components of the plasma nozzle described in the general process description did not reveal any visible extensive corrosion on their surface (as 
shown in Figure 14). The choices made in terms of geometry, cooling and materials were therefore validated.
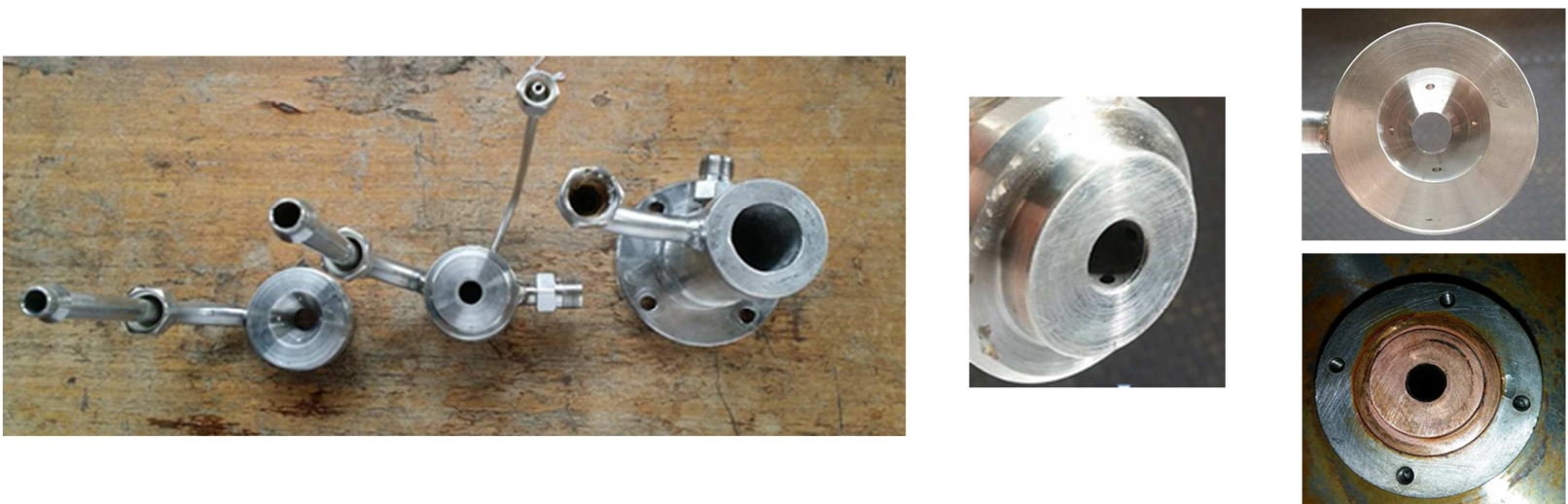

Figure 14: Appearance of torch outlet nozzle components following treatment of TBP/Dodecan, TCE and PFPE liquids at a rate of $2 \mathrm{~L}^{-1}{ }^{-1}$.

This evaluation demonstrates an excellent response by the process for treatment of the liquids mentioned above. The effective destruction rates obtained in these initial experiments was better than $99 \%$, and may be improved by certain upgrades that are currently being studied.

Additional tests that are not covered in the present article were also conducted to test the treatment of TBP/Dodecan at rates of up to $5 \mathrm{~L} \cdot \mathrm{h}^{-1}$. The results currently show effective destruction rates that remain above $99 \%$, with no or very little corrosion.

\section{CONCLUSION}

The ELIPSE process is an innovative and promising solution for the destruction of radioactive organic liquids. The simplicity of the concept and its apparent robustness offer reasons to believe that it may be used to treat a variety of liquid organic-containing wastes.

The innovative feature of this process is that it uses an arc plasma torch submerged in an aqueous solution. The plasma torch, in which the organic liquids are injected with no 
previous dilution, affords rapid and almost complete destruction of the molecules. The aqueous solution guarantees that the combustion gases are treated by ensuring their cooling, filtering and cleaning. The gas treatment function is then transferred to a treatment system for an aqueous solution with a density that is a thousand times greater than that of a gaseous phase. This explains why it is possible for the system to be extremely compact, as the treatment system for the gases is in fact limited to a simple condenser, with a safety filter downstream to prevent any escape to the stack.

The use of an aqueous solution that is continuously filtered and cooled via a heat exchanger ensures that the entire process remains cooled. This robust design is extremely flexible and the resulting process may be used to treat a wide variety of liquids containing chlorine, fluorine, phosphorous, sulfur, etc. as many corrosion problems can be controlled by cooling. The treatment system for the solution, which ensures filtering amongst other functions, makes it possible to collect the insoluble inorganic constituants that were initially contained in the organic liquids, including insoluble radioactive species and phases. The resulting inorganic phases can be stabilized by cementation or vitrification.

The component most susceptible to corrosion is the torch nozzle. Its walls are continuously cooled by an internal cooling water circulation system to ensure acceptable corrosion resistance and no significant corrosion was observed during testing.

These initial tests performed on ELIPSE for the destruction of trichloroethylene (with high chlorine content), a TriButylPhoshate-Dodecane mixture (with high phosphorus content) and a perfluorinated oil (with high fluorine content) have indicated destruction levels equal to or greater than $99.8 \%$ with no special optimizations. These results offer major prospects for the application at the currently accessible processing rate of $5 \mathrm{~L} \cdot \mathrm{h}^{-1}$ on the pilot test facility, which could be significantly increased based on treatment needs with the use of more powerful plasma torch based on treatment needs. 
Although the obtained results are already promising enough to justify an industrialization study for the system, optimization work has been undertaken to improve the obtained destruction levels. These optimizations concerned the geometry and the operational mode of the plasma torch nozzle, and the use of the radiation emitted by the plasma to activate advanced oxidation reactions in the core of the solution. In this last case the current studies are now showing the capability of the submerged UV radiation to produced hydrogen peroxide in the solution. Thus, it could be possible to trigger a photo Fenton reaction (Figure 15) in the core of the aqueous solution in order to degrade the residual organic matter passing through the solution. These optimizations should make it possible over the short term to obtain destruction levels greater than $99.9 \%$. This will be published in a upcoming paper.

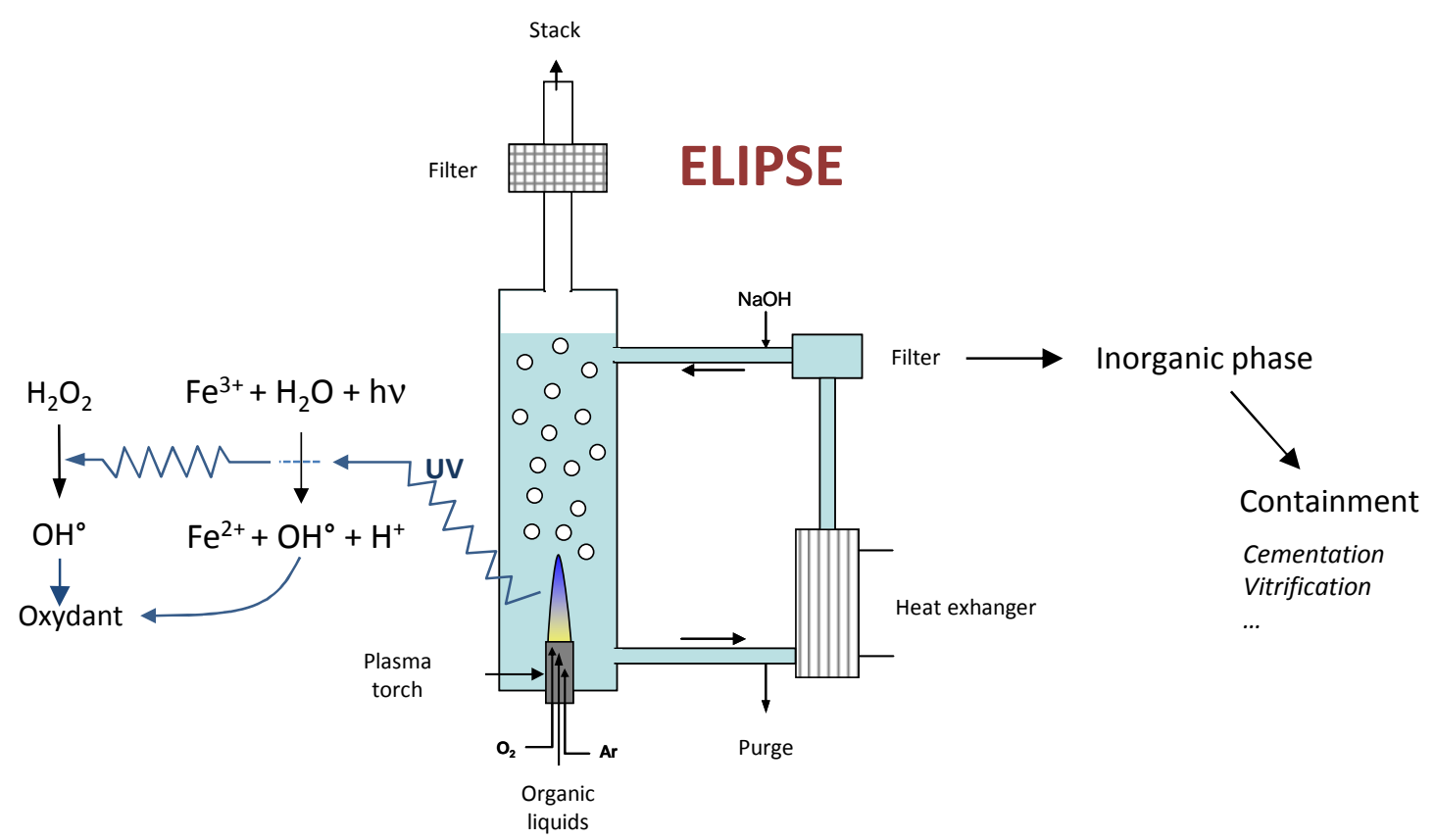

Figure 15: ELIPSE process optimized by use of a photo-activated Fenton reaction in core of aqueous solution. 


\section{REFERENCES}

[1] Estimation of Global Inventories of Radioactive Waste and Other Radioactive Materials. IAEA-TECDOC-1591 - June 2007

[2] Shuey M.W., Ottmer P.P., LLW Processing and Operational Experience using Plasma ARC Centrifugal treatment (PACT $\left.{ }^{\mathrm{TM}}\right)$ System. WM'06 Conference, February 26-March 2, 2006, Tucson AZ - USA

[3] Redonnet C., Runge S., Moulin J.P., MDS: A proven and versatile solvent mineralization process, WM'00 Conference, February 27 - March 2, 2000, Tucson AZ - USA

[4] Luycx P., Deckers J, Pebble bed pyrolysis for the processing of alpha contaminated organic effluents, WM'99 Conference, February 28 - March 4, 1999, Tucson AZ - USA

[5] LWW Repository Ltd, National Waste Programme, Management of Contaminated Oils, Feasibility Study. NWP-REP-086 - Issue 1 - Mars 2015

[6] Y.Wu, L.M. Polvani, R.Seager, The importance of the Montreal Protocol in Protecting Earth's Hydroclimate, http://dx.doi.org/10.1175/JCLI-D-12-00675.1

[7] Shuey M.W., Ottmer P.P., LLW Processing and Operational Experience using a Plasma ARC Centrifugal Treatment $\left(\mathrm{PACT}^{\mathrm{TM}}\right)$ System, WM'06 Conference, February 26 - March 2, 2006, Tucson AZ - USA

[8] Lemont F., Charvin P., Russello A., Poizot K., An Innovative Hybrid Process Involving Plasma in a Cold Crucible Melter Devoted to the Future Intermediate Level Waste Treatment: The SHIVA Technology. Advances in Sciences and Technology Vol.73. pp 148157,2010

[9] Deam RT., Briar H, Kearney TN. Material processing. EP Patent 0629138:B1 
[10] Alekseev NV., Pozdnyakov OE., Shorin SN., Study of the interaction between a hot gas jet and a liquid bath. J Eng Phys Thermophys 44(4). pp 358-363, 1983

[11] Alekseev NV.,Samokhin AV., Belivtsev AN., Zhavoronkova VI., Thermal-plasma jet oxidation of phenol in aqueous solutions. High Energy Chem 34(6). pp 389-393, 2000

[12] Bernier JL., Fortin L., Kimmerle FM., Boulos MI. Kasireddy V., Soucy G.. Thermal plasma reactor and wastewater treatment method. Patent - WO97/22556- 1997.

[13] Uchiyama G., Maeda M., Fujine S., Amakawa M., Uchida K., Chida M. Development of spent solvent treatment process by a submerged combustion technique. Journal of Nuclear Science and Technology. 31(3) pp.228-239. 1994

[14] Nakagama A., Sone T., Sasaki T, Nakazawa O., Tashiro K., Performance of Steam Reforming Technology in a Long Term Treatment of Waste TBP/Dodecane. WM2011 Conference, February 27 - March 3, 2011, Phoenix, AZ

[15] IAEA. Predisposal management of organic radioactive waste. Technical reports series №.427. International Atomic Energy Agency, 2004, V ienna

[16] Holleman A.F., N.Wiberg, Inorganic Chemistry, Academic Press, 2001

[17] A.D.Starostin, A.V. Nikolaev, Y.A. Afans'ev. Standard heats of formation of certain organophosphorus compounds. Bulletin of the Academy of Sciences of the USSR, Division of chemical science. August 1966, V15, 18 pp 1255-1258 